\title{
A test of the chronic-acute hypothesis in semantic GSR conditioning
}

WOLFGANG G. BRINGMANN

UNIVERSITY OF MISSISSIPPI

Two alternative hypotheses (chronic vs. acute) about the nature of humin anxiety, its relation to intensity of noxious stimulation, and its energizing effect in simple learning situations were examined. One group of $S s$ with high and one with low scores on a manifest anxiety scale $\left(\mathrm{N}_{-} 38\right)$ were conditioned to give a GSR to the CS "light", with a $500 \mathrm{cps}$ tone at $70 \mathrm{~dB}$ serving as non-noxious UCS. A tone of 500 cps at $90 \mathrm{~dB}$ served as noxious UCS for the other anxious and non-anxious groups $(\mathrm{N}=37)$. A significant interaction between anxiety and UCS intensity obtained both for generalization and for extinction measures. The results support the acute hypothesis which states that high anxiety scores reflect an "acute" rather than a chronic state of anxiety.

Recently Spence and his associates successfully demonstrated the energizing function of anxiety in basic human learning (Brown, 1961). In most studies at Iowa, different levels of anxiety were responsedefined by the selection of Ss with extreme scores on a self-report inventory (Taylor, 1953) - the Manifest Anxiety Scale (MAS). A smaller number of experiments is reported in which anxiety was stimulusdefined through the introduction of stress in the actual learning situation. Although a substantial body of knowledge, concerning the effects of either high MAS scores or noxious stimulation upon performance, exists, not much has been known concerning the interaction between those two variables.

Two hypotheses have been offered by Spence (1956) regarding the nature of anxiety, measured by the MAS, and its relation to environmental stimulation. According to the first of these views (chronic hypothesis), anxiety is regarded as a trait which is chronically present in certain individuals. The other position (acute hypothesis) views anxiety as situational, as an acute reaction to stressful stimulation. Since experimental evidence bearing on these rival interpretations is meager and inconclusive (Brown, 1961), it was the chief purpose of the present study to critically examine once more the validity of the two theories.

To overcome limitations of previous tests of the chronic-acute issue, several methodological innovations were introduced with respect to the experimental task, the procedures for varying stress experimentally, the performance measures, and the criteria of anxiety (Bringmann, 1964). Anxiety scores and intensity of a tonal UCS were independent variables. Generalization and extinction scores were selected as measures of the dependent variable because of their relative neglect in previous tests of the chronic- acute hypothesis (Bringmann, 1964) and their importance in other studies dealing with the energizing effect of anxiety (Kimble, 1961). A simple factorial design with two levels of anxiety (high and low) and two levels of UCS intensity (weak and strong) utilized four experimental groups. According to the chronic hypothesis, an interaction between anxiety scores and UCS intensity should not result. The acute hypothesis would predict a reliable interaction, i.e., the high anxious groups should separate more strongly from the low anxious groups under high stress (strong tonal UCS) than under weak stress (weak tonal UCS). Subjects

The experimental population consisted of 75 nursing students chosen from introductory psychology classes on the basis of extreme scores on the Heinemann Forced Choice Manifest Anxiety Scale (Heinemann, 1953). Two high anxious groups consisted of $38 \mathrm{Ss}$ scoring within the upper 20 percent on the HFC-MAS. Thirty-seven Ss, scoring within the lower 20 percent on the anxiety scale, were designated as the low anxious groups.

\section{Apparafus}

Galvanic Skin response measures were made by a Grason-Stadler Psychogalvanometer (Model E-664).

A $500 \mathrm{cps}, 5$ percent warble tone, $70 \mathrm{~dB}$ above individual loudness thresholds, served as non-noxious UCS. The same tone presented at an intensity level of $90 \mathrm{~dB}$ constituted the noxious UCS.

A Kodak Carousel Projector (Model 550) presented the stimulus words.

All verbal stimuli were chosen from the KentRosanoff Word Association Test by an empirical method developed by Mednick (1957). The word "light" was used as CS because it is frequently given as association to the generalization test words "dark, heavy, lamp, and soft." The neutral test word "square" and 14 filler words were added to the word lists because they showed no empirical relation to the CS.

The experiment was conducted in an auditory testing unit furnished with a projection screen and a comfortable chair.

\section{Procedure}

Each $\mathrm{S}$ was attached to the equipment by an assistant and instructed to read in normal speaking voice the words shown on the screen.

The CS and the filler words were shown twice in random sequence but without reinforcement during the preliminary phase of the study. Between the first and second projection of this word, the UCS for each 
$S$ was delivered three times while the screen remained dark. This procedure was employed as pseudoconditioning control and to evaluate the effectiveness of the stress method.

During the immediately following conditioning phase, four conditioning word lists each consisting of the filler words and six presentations of the CS were shown. Reinforcement was delivered randomly 50 percent of the time to obtain stronger conditioning and to use the trials without UCS as tests for conditioning.

The CS, the generalization words, and the neutral test words were repeated six times in random order during the generalization-extinction stage of the experiment but without reinforcement. The first repetition was considered a test of semantic generalization.

Before dismissal each $S$ was given a brief interview including the request to rate her respective UCS as either "pleasant, neutral, or unpleasant."

Statistics.

An individual was considered conditioned when two or three consecutive presentations of the CS alone elicited a GSR greater than that accompanying the last filler word immediately preceding the CS. Resistance data obtained directly from GSR tracings were transformed into conductance units and subjected to variance analysis (Lacey \& Siegel, 1949).

\section{Results}

Generalization. Mean GSR amplitudes in response to the CS, tne generalization stimuli, and the neutral test word are shown in Table 1. Differences in generalization among the four experimental groups are quite striking. Inspection of Table 1 reveals that GSR responsiveness during the generalization phase appears to conform to a gradient defined by the degree to which each of the generalization words was associatively related to the conditioned stimulus in Mednick's (1957) experiment. Results of an analysis of variance of the total GSR amplitudes during generalization clearly support the acute hypothesis, i.e., the interaction between anxiety level and UCS intensity is seen to be highly significant ( $F=9.80, d f=1 / 64, p<.01$ ).

Extinction. Mean GSR amplitudes in response to the CS "light" during each of the six extinction series are summarized in Table 2. Once more high anxious groups demonstrate considerably more re-

Table 1. Mean GSR Amplitudes in Micromhos for Each Word During The Generalization (First Extinction) Series

\begin{tabular}{lccccccc} 
Groups & N & Light & Lamp & Dark & Heovy & Soft & Square \\
\hline HAWT & 17 & 1.02 & 0.67 & 0.60 & 0.53 & 0.51 & 0.12 \\
HAST & 20 & 1.27 & 0.95 & 0.89 & 0.68 & 0.46 & 0.16 \\
LAWT & 15 & 0.75 & 0.57 & 0.52 & 0.33 & 0.38 & 0.12 \\
LAST & 16 & 0.84 & 0.60 & 0.59 & 0.48 & 0.31 & 0.08 \\
\hline
\end{tabular}

Note-The following abbreviations describe the four experimental groups on Table 1 and 2: HAWT (High Anxiety, Weak Tone). HAS'T (High Anxzety, Strong Tone), LAWT (Low Anxiety, Weak Tone), and LAST (Low Anxiety, Strong Tone).
Table 2. Mean GSR Amplitudes in Micromhos for CS "LIGHT" During Extinction

\begin{tabular}{lccccccc} 
Groups & N & 1 & 2 & 3 & 4 & 5 & 6 \\
\hline HAWT & 17 & 1.02 & 0.78 & 0.45 & 0.30 & 0.44 & 0.33 \\
HAST & 20 & 1.27 & 0.84 & 1.03 & 0.68 & 0.51 & 0.48 \\
LAWT & 15 & 0.75 & 0.50 & 0.42 & 0.24 & 0.10 & 0.00 \\
LAST & 16 & 0.84 & 0.65 & 0.47 & 0.25 & 0.00 & 0.00 \\
\hline
\end{tabular}

sponsiveness throughout extinction than low anxious groups. An analysis of variance of total GSR amplitudes during extinction yields, in agreement with the acute hypothesis, a significant interaction effect $(F=4.70, d f=1 / 64, p<.05)$.

\section{Discussion}

The present study stands alone in finding interaction between anxiety scores and UCS intensity in semantic GSR conditioning. However, one study of simple GSR conditioning (Beam, 1955) and one of eyelid conditioning (Sweetbaum, 1963) have yielded comparable results.

The significant interaction between anxiety scores and UCS intensity of this study contributes towards a solution of the chronic-acute problem by suggesting that anxiety, as measured by the HFC-MAS and the MAS, is largely an "acute" reaction to different levels of stress. Such an acute conception of the nature of anxiety agrees well with clinical experience and recent experimental formulations (Endler \& Hunt, 1966; Spence, 1964).

\section{References}

Beam, J. C. Serial learning and conditioning under real life stress. J. abnorm. soc. Psychol., 1955, 51, 543-551.

Bringmann, W. G. The effects of anxiety and stimulus intensity on performance in semantic GSR conditioning: A test of the chronicacute hypothesis. Unpublished doctoral dissertation, University of Alabama, 1964.

Brown, J. S. The motivation of behavior. New York: McGraw-Hill, 1961.

Endler, N. S., \& Hunt, J. McV. Sources of behavioral variance as measured by S-R inventory of anxiousness. Psychol. Bull., $1966,65,336-346$.

Heinemann, C. E. A forced choice form of the Taylor manifest anxiety scale, J. consult. Psychol., 1953, 17, 447-454.

Kimble, G. A. Conditioning and learning. (Rev. ed.) New York: Appleton, 1961 .

Lacey, O. L., \& Siegel, P. S. An analysis of the unit of measurement of the galvanic skin response. J. exp. Psychol., 1949, $39,122-127$.

Mednick, Martha T. Mediated generalization and the incubation effect as a function of manifest anxiety. J. abnorm. soc. Psychol., 1957, 55, 315-326.

Spence, K. W. Behavior theory and conditioning. New Haven: Yale University Press, 1956.

Spence, K. W. Anxiety (drive) level and performance in eyelid conditioning. Psychol. Bull., 1964, 129-139.

Sweetbaum, H. A. Comparisons of the effects of introversionextroversion and anxiety on conditioning. J. abnorm. soc. Psychol., 1963, 66, 249-254.

Taylor, Janet A. A personality scale of manifest anxiety. J. $a b-$ norm. soc. Psychol., 1953, 48, 285-290. 